\title{
Robust Student's T Distribution Based PHD/CPHD Filter for Multiple Targets Tracking Using Variational Bayesian Approach
}

\author{
Peng $L I^{1}$, Chen $X U^{2}$, Wenhui WANG ${ }^{1}$, Shuzhi $S U^{3}$ \\ ${ }^{1}$ School of Computer Engineering, Jiangsu University of Technology, Changzhou, 213001, China \\ ${ }^{2}$ School of Internet of Things Engineering, Jiangnan University, Wuxi, 214122, China \\ ${ }^{3}$ College of Computer Science and Engineering, Anhui University of Science \& Technology, Huainan, 232001, China
}

lipengjiangnan@163.com, lpjsut@foxmail.com,wangmanwenhui@163.com, sushuzhi@foxmail.com

Submitted December 24, 2019 / Accepted June 11, 2020

\begin{abstract}
Measurement-outliers caused by non-linear observation model or random disturbance will lead to the accuracy decline of a target tracking filter. This paper proposes a robust probability hypothesis density (PHD) filter to handle the measurement-outlier problem based on Student's T Kalman (TK) filtering technique and Variational Bayesian (VB) method. First, the non-standard measurement noise is considered to follow the Student's T distribution. Second, the TK filtering technique is employed to update the target states. Third, the posterior likelihood is updated by the VB approach. Simulation results show that the proposed method can reduce the optimal subpattern assignment (OSPA) error in the non-standard observation scenarios with measurement-outliers, compared with other typical multiple target tracking filters.
\end{abstract}

\section{Keywords}

Multiple target tracking, PHD filter, Student's T Kalman, Variational Bayesian, non-linear filter

\section{Introduction}

Multiple targets tracking has important applications in sonar, navigation, radar detection and other engineering fields. Based on Finite Set Statistics (FISST), Mahler proposed the random finite set (RFS) observation model [1]. The first-order moment of an RFS is the PHD [1], which is an intensity function defined over the state space. Another important observation model is the labeled random finite set (LRFS) [2], which establishes a strict mathematical model for track association problem. The typical implementations of the LRFS theory are generalized labeled multi-Bernoulli (GLMB) filter [3] and labeled multi-Bernoulli (LMB) filter [4]. Up to now, the PHD filters and GLMB/LMB filters are the hottest topic in multiple targets tracking field.
In the RFS theory, one of the most important implementations of the PHD framework is the Gaussian mixture PHD (GM-PHD) filter [5], which assumes that the measurement noise follows the Gaussian distribution, therefore the PHD can be approximated by a Gaussian mixture form. In [6] and [7], Mahler derived the cardinality distribution and presented a cardinalized PHD (CPHD) filter. The CPHD filter can provide higher-order estimations on the number of targets, which leads to better tracking performance compared with the PHD filter. Since it expenses higher computational costs, researchers modified the CPHD filter in [8-10]. However, the PHD/CPHD filters cannot provide track estimates due to target tracks of a RFS filter are not ordered. To solve this problem, Clark et al. developed a weight-based track management scheme for GM-PHD filter in [11], and Panta et al. presented a novel tree-structure-based track management scheme to track crossing targets in [12]. However, these schemes are only supplements to the RFS theory and are not based on strict mathematical derivation.

The LRFS theory was presented by Vo et al. to solve the problem that the RFS theory does not establish a strict mathematical model for target trajectory [2]. Based on LRFS, Vo et al. presented the delta GLMB ( $\delta$-GLMB) filter [2] and Reuter et al. presented the LMB filter [3]. The GLMB and LMB approaches have high accuracy in state estimation due to they can formally estimate tracks. However, they still have the problem of high computational complexity. Thus, a joint GLMB/LMB filter was proposed to reduce the computational complexity in [13]. The truncation procedures are modified by the Gibbs sampling method and a joint prediction and update method is used to eliminate inefficiencies, with further modifications in [14]. Recently, a modified GLMB filter was presented in [15], which can handle the signal-to-noise ratio scenarios.

For non-standard measurement scenarios, the particle, extended Kalman (EK) [16] and unscented Kalman (UK) [17] filtering techniques are commonly used for RFS and 
LRFS tracking approaches. The Sequential Monte Carlo PHD (SMC-PHD) filter [18] is a typically particle implementation of PHD filter to handle non-linear scenarios. However, the SMC-PHD filter takes a lot of computational costs, which limits its practical application. In [4] and [19], EK, UK and particle implementations were utilized in the GM-PHD filter to handle the bearing and range (BAR) measurement scenarios. Recently, an LMB filter for bearings-only scenarios was presented in [20]. In [21], an improved measurement-oriented marginal multi-Bernoulli/ Poisson (IMOMB/P) filter was presented to solve the measurement miss detection problem. In [22], a multimodel particle filter was presented to handle the maneuvering target.

In recent years, Student's T distribution is considered to be more robust than Gaussian distribution in non-standard scenarios, thus it has become a hot topic in target tracking. Huang et al. presented a robust Student's T Kalman filter in [23] to handle the heavy-tailed measurement filtering problems. In [24], a Gaussian-Student's T mixture distribution Kalman filter was proposed. This filter uses the VB based TK filtering method [26] and the inverse Wishart distribution to jointly infer the state vector, auxiliary random variables, Bernoulli random variables and mixing probabilities. A Student's T distribution based PHD filter for multiple target tracking was proposed in [25]. In this filter, the measurement likelihood is derived by the Student's $T$ distribution instead of Gaussian distribution, thus its performance is better than the GM-PHD filter in scenarios with measurement-outliers. However, this work does not consider the application of VB technology to improve the robustness.

This paper based on the VB based TK filtering technique in [26] proposes the robust Student's T Kalman PHD/CPHD (TK-PHD/CPHD) filters and extended TKPHD (E-TK-PHD) filter to handle the measurement-outliers and BAR scenarios. The code is published on GitHub [31]. The contributions are as follows:

1) The TK filtering technique [26] and VB framework [27] are employed to update the posterior intensity each scan. Therefore, the proposed TK-PHD and TKCPHD filters can converge quickly through iteration to improve tracking accuracy when outliers are generated, and have higher robustness in non-linear scenarios.

2) The E-TK-PHD implementation was proposed for BAR scenarios. It uses the idea of solving Jacobian matrix in extended Kalman filter technique to deal with BAR scenarios, thus its accuracy is higher than linear implementations in BAR scenarios.

The paper is organized as follows. Section 2 introduces the VB approach and TK filter. The implementation methods of the TK-PHD/CPHD and E-TK-PHD filters for measurement-outliers and BAR scenarios are presented in Sec. 3. Section 4 shows the simulation results. Section 5 contains our conclusions.

\section{Background}

\subsection{The Variational Bayesian Inference}

Assuming that the true posterior probability density is $g(Z \mid X)$, the key step of the VB approach is to use an approximate posterior probability density $\Psi(Z)$ to approximate $g(Z \mid X)$. According to [27], a distribution can be written as sum of two parts, i.e.

$$
\begin{aligned}
\log P(X)= & \underbrace{\int q(z) \log P(x, z) \mathrm{d} z-\int q(z) \log q(z) \mathrm{d} z}_{L(q(z)), E L O B(\text { Evidence Lower Bound })},(1) \\
& +\underbrace{\int q(z) \log \frac{q(z)}{P(z \mid x)} \mathrm{d} z}_{K L(q(z) \mid P(z \mid x))}
\end{aligned}
$$

where $L(\cdot)$ is called ELOB, $K L(\cdot \mid \cdot)$ is called $\mathrm{KL}$ divergence [28], which denotes the distance between two distributions. Therefore, the new form of $\Psi(Z)$ and $g(Z \mid X)$ can be obtained by

$$
\log g(X)=K L(\Psi \| g)+L(\Psi) .
$$

The problem translates into minimizing $K L(\Psi \| g)$. Assuming that $g(X)$ is constant, $K L(\Psi \| g)$ can be minimized by maximizing $L(\Psi)$. Assuming that each hidden variable obeys an independent and identical distribution, thus $\Psi(Z)=\prod_{i} \Psi\left(z_{i}\right) . L(\Psi)$ can be rewritten by

$$
\begin{aligned}
L(\Psi)= & -K L\left(\Psi\left(z_{j}\right) \| \Psi^{*}\left(z_{j}\right)\right) \\
& +\prod_{i: i \neq j} H\left(\Psi\left(z_{i}\right)\right)-\mathrm{const} .
\end{aligned}
$$

Note that $z_{i}$ and $z_{j}$ are the single variable form divided by complex variable $Z$. $i$ and $j$ are the subscript of the information entropy $\prod_{i: i \neq j} H\left(\Psi\left(z_{i}\right)\right)$ and KL divergence. Obviously, $\prod_{i: i \neq j} H\left(\Psi\left(z_{i}\right)\right) \geq 0$ and the value of KL divergence is larger than zero as well. According to (2), our goal is to maximize $L(\Psi)$. The information entropy is not constant, thus $L(\Psi)$ will maximize when $-\operatorname{KL}\left(\Psi\left(z_{j}\right) \| \Psi^{*}\left(z_{j}\right)\right)=0$. Therefore, $\Psi\left(z_{j}\right)$ can be obtained by

$$
\Psi\left(z_{j}\right)=\Psi^{*}\left(z_{j}\right)=\frac{\exp \left(E_{i \neq j}[\log g(Z, X)]\right)}{\text { normalize constant }} .
$$

By way of a given number times recurrences, $\Psi\left(z_{j}\right)$ can be approximated to $g(Z \mid X)$.

\subsection{The VB Based Student-T Kalman Filter}

The TK filter was presented in [26], which can deal with non-linear measurement models, especially measure- 
ment-outlier models. The observation model with Student's $\mathrm{T}$ distributed measurement noise is

$$
\begin{gathered}
x_{k} \mid x_{k-1} \sim \mathrm{N}\left(f\left(x_{k-1}\right), \mathbf{Q}_{k}\right), \\
z_{k} \mid x_{k} \sim \operatorname{St}\left(h\left(x_{k}\right), \mathbf{R}_{k}, v\right)
\end{gathered}
$$

where $x_{k}$ is the state. $z_{k}$ is the measurement. $\operatorname{St}(\cdot)$ is the Students' $\mathrm{T}$ distribution. $f(\cdot)$ and $h(\cdot)$ are dynamic and measurement model functions, $v$ is the degree of freedom. $\mathbf{Q}_{k}$ and $\mathbf{R}_{k}$ are covariance matrices of process and measurement noise, respectively. According to [26], the Student's $\mathrm{T}$ distribution's probability density is

$$
\begin{aligned}
\operatorname{St}\left(h\left(x_{k}\right), \mathbf{R}_{k}, v\right) & \propto\left(1+\frac{1}{v}\left(z_{k}-h\left(x_{k}\right)\right)^{\mathrm{T}}\right. \\
& \left.\times \mathbf{R}_{k}^{-1}\left(z_{k}-h\left(x_{k}\right)\right)\right)^{-\frac{v+d}{2}}
\end{aligned}
$$

where $\operatorname{St}(\cdot)$ denotes Student's T distribution, $d$ is the dimensions of physical space. When $v \rightarrow 0$, the above Student's T distribution converges to $\mathrm{N}\left(h\left(x_{k}\right), \mathbf{R}_{k}\right)$. The TK filter employs an auxiliary random variable $\lambda_{k}$, thus the likelihood can be approximately expressed as a hierarchical Gaussian forms, i.e. (5b) can be rewritten as

$$
\begin{gathered}
z_{k} \mid x_{k}, \lambda_{k} \sim \mathrm{N}\left(h\left(x_{k}\right), \frac{1}{\lambda_{k}} \mathbf{R}_{k}\right), \\
\lambda_{k} \sim \operatorname{Gamma}(v / 2, v / 2) .
\end{gathered}
$$

Therefore, the update steps of the VB based TK filter can be approximately implemented as follows:

$$
\begin{gathered}
\mathbf{m}_{k}=\mathbf{m}_{k \mid k-1}+\mathbf{K}_{k}\left(z_{k}-\mu_{k}\right), \\
\mathbf{P}_{k}=\mathbf{P}_{k \mid k-1}+\mathbf{K}_{k} \mathbf{S}_{k} \mathbf{K}_{k}^{\mathrm{T}},
\end{gathered}
$$

where

$$
\begin{gathered}
\boldsymbol{\mu}_{k}=\int h\left(x_{k}\right) \mathrm{N}\left(x_{k} \mid \mathbf{m}_{k \mid k-1}, \mathbf{P}_{k \mid k-1}\right) \mathrm{d} x_{k}, \\
S_{k}=\int\left(h\left(x_{k}\right)-\boldsymbol{\mu}_{k}\right)\left(h\left(x_{k}\right)-\boldsymbol{\mu}_{k}\right)^{\mathrm{T}} \\
\times \mathrm{N}\left(x_{k} \mid \mathbf{m}_{k \mid k-1}, \mathbf{P}_{k \mid k-1}\right) \mathrm{d} x_{k}+\frac{1}{\lambda_{k}} \mathbf{R}_{k}, \\
K_{k}=\int\left(x_{k}-\mathbf{m}_{k \mid k-1}\right)\left(h\left(x_{k}\right)-\boldsymbol{\mu}_{k}\right)^{\mathrm{T}} \\
\times \mathrm{N}\left(x_{k} \mid \mathbf{m}_{k \mid k-1}, \mathbf{P}_{k \mid k-1}\right) \mathrm{d} x_{k} \cdot \mathbf{S}_{k}^{-1}, \\
\bar{\gamma}_{k}=\operatorname{tr}\left(\int\left(z_{k}-h\left(x_{k}\right)\right)\left(z_{k}-h\left(x_{k}\right)\right)^{\mathrm{T}}\right. \\
\left.\times \mathrm{N}\left(x_{k} \mid \mathbf{m}_{k}, \mathbf{P}_{k}\right) \mathrm{d} x_{k} \mathbf{R}_{k}^{-1}\right)
\end{gathered}
$$

where $\operatorname{tr}(\cdot)$ denotes the trace of a matrix. Through a given number of $N_{k}$ times recurrences, $\lambda_{k}$ converges.

\subsection{The PHD Filter}

A PHD filter is an approximation to solve the computational problem of the multi-target Bayesian filter. Let $D_{k}$ and $D_{k \mid k-1}$ denote the intensities of the posterior and predicted density respectively, the target PHD is

$$
\begin{aligned}
D_{k \mid k-1}(x) & =\int p_{S, k}(\zeta) f_{k \mid k-1}(x \mid \zeta) D_{k-1}(\zeta) \mathrm{d} \zeta \\
& +\int \beta_{k \mid k-1}(x \mid \zeta) D_{k-1}(\zeta) \mathrm{d} \zeta+\vartheta_{k}(x), \\
D_{k}(x)= & {\left[1-p_{D, k}(x)\right] D_{k \mid k-1} } \\
+ & \sum_{z \in Z_{k}} \frac{p_{D, k}(x) g_{k}(z \mid x) D_{k \mid k-1}(x)}{\kappa_{k}(z)+\int p_{D, k}(\xi) g_{k}(z \mid \xi) D_{k \mid k-1}(\xi) \mathrm{d} \xi}
\end{aligned}
$$

where $\zeta$ and $\xi$ denote target prior and posterior states. $z$ denotes the measurement. $\beta_{k \mid k-1}(\cdot)$ denotes the target spawning intensity, and $\vartheta_{k}(x)$ denotes the target birth intensity. $p_{S, k}(\cdot)$ and $p_{D, k}(\cdot)$ are target existing and detection probabilities. $\kappa_{k}(z)$ is the clutter intensity. $g_{k}(z \mid x)$ is the multi-target likelihood.

The PHD filter use $D_{k \mid k-1}(x)$ and $D_{k}(x)$ to calculate the weight of each component, and the components with large weights are considered targets. The existing PHD framework mainly use the Kalman filter to update target states, and use Gaussian mixture or particle filtering approaches to approximate the likelihood $g_{k}(z \mid x)$. The contribution of this work is to replace Kalman filter with TK filter, and propose a new likelihood. Therefore, the PHD filter will be robust to measurement-outliers.

\section{The Student's T Kalman PHD Filter}

\subsection{Key Method}

The updating step of a PHD filter is a multiple hypothesis process between the prediction components and all the measurements each scan. The state of each prediction component will be updated by a corresponding measurement, and generates a posterior components. Typically, the components are Gaussian mixture form and the measurements are assumed to follow the Gaussian distribution, i.e., the likelihood $g_{k}(z \mid x)$ in $(10 \mathrm{~b})$ is the Gaussian probability density. Moreover, the Gaussian form is suitable for the Kalman filtering technology, thus Kalman filter is usually used in a PHD filter. However, the Gaussian distribution is only an ideal case, which leads to a lack of robustness of a standard PHD filter.

Compared with the Gaussian distribution, the Student's T distribution has stronger robustness. Our key method is to find a robust way to replace Gaussian model with Student's T model. Our first work is to employ the more robust TK filtering technology to improve the state 
updating process. However, the TK filter is based on VB technology, thus the likelihood $g_{k}(z \mid x)$ cannot be assumed as the Student's T density directly. Therefore, our second work is to derive a new likelihood function for the PHD filter using KL divergence, i.e. formulas (22)-(32).

\subsection{The Iteration of TK-PHD Filter}

The TK filter has strong robustness and accuracy in the outlier scenarios. Therefore, the key method of this work is to use TK filter to improve the state updating step of a PHD filter and approximate the weights of components. Assuming that the state space system is given by (5), the target predicted PHD is the same as (13a).

According to [26], in order to implement the TK filter, an auxiliary random variable $\lambda_{k}$ was introduced. Based on (5b), (7) and (8), the posterior PHD can be rewritten as

$$
\begin{aligned}
& D_{k}(x)=\left[1-p_{D, k}(x)\right] D_{k \mid k-1} \\
& +\sum_{z \in Z_{k}} \frac{p_{D, k}(x) \Psi_{k}\left(z \mid x, \lambda_{k}\right) D_{k \mid k-1}(x)}{\kappa_{k}(z)+\int p_{D, k}(\xi) \Psi_{k}\left(z \mid x, \lambda_{k}\right) D_{k \mid k-1}(\xi) \mathrm{d} \xi}
\end{aligned}
$$

where $\lambda_{k} \sim \operatorname{Gamma}(v / 2, v / 2)$. The most important contribution is to replace (10b) with (11), and to derive the specific updating method of the new $D_{k}(x)$.

\subsection{The Implementation for Measurement- Outlier Scenarios}

Assuming that the state space system is given by (5), in this work, the target prediction PHD is equal to the standard GM-PHD filter [4], and can be obtained by

$$
D_{k \mid k-1}(x)=D_{S, k \mid k-1}(x)+D_{\beta, k \mid k-1}(x)+\vartheta_{k}(x)
$$

where $D_{S, k \mid k-1}(x), D_{\beta, k \mid k-1}(x)$ and $\vartheta_{k}(x)$ denote the predicted intensity of existing, spawning and birth targets, respectively. $D_{S, k \mid k-1}(x)$ is given by

$$
\begin{gathered}
D_{S, k \mid k-1}(x)=p_{S, k} \sum_{j=1}^{J_{k-1}} w_{k-1}^{(j)} \mathrm{N}\left(x \mid \mathbf{m}_{S, k \mid k-1}^{(j)}, \mathbf{P}_{S, k \mid k-1}^{(j)}\right), \\
\mathbf{m}_{S, k \mid k-1}^{(j)}=\mathbf{F}_{k-1} \mathbf{m}_{k-1}^{(j)} \\
\mathbf{P}_{S, k \mid k-1}^{(j)}=\mathbf{Q}_{k-1}+\mathbf{F}_{k-1} \mathbf{P}_{k-1}^{(j)} \mathbf{F}_{k-1}^{\mathrm{T}}
\end{gathered}
$$

where $\mathbf{F}_{k-1}$ is the state transition matrix. $\mathbf{m}_{k-1}^{(j)}$ and $\mathbf{P}_{k-1}^{(j)}$ are the kinematical state and covariance matrix of the $j$ th component at time $k-1 . D_{\beta, k \mid k-1}(x)$ is given by

$$
\begin{gathered}
D_{\beta, k \mid k-1}(x)=\sum_{j=1}^{J_{k-1}} \sum_{\ell=1}^{J_{\beta, k}} w_{k-1}^{(j)} w_{\beta, k}^{(\ell)} \mathrm{N}\left(x \mid \mathbf{m}_{\beta, k \mid k-1}^{(j, \ell)}, \mathbf{P}_{\beta, k \mid k-1}^{(j, \ell)}\right), \\
\mathbf{m}_{\beta, k \mid k-1}^{(j, \ell)}=\mathbf{F}_{\beta, k-1}^{(\ell)} \mathbf{m}_{k-1}^{(j)}+\mathbf{d}_{\beta, k-1}^{(\ell)}, \\
\mathbf{P}_{\beta, k \mid k-1}^{(j, \ell)}=\mathbf{Q}_{\beta, k-1}^{(\ell)}+\mathbf{F}_{\beta, k-1}^{(\ell)} \mathbf{P}_{\beta, k-1}^{(j)}\left(\mathbf{F}_{\beta, k-1}^{(\ell)}\right)^{\mathrm{T}}
\end{gathered}
$$

The birth intensity $\vartheta_{k}(x)$ is given by

$$
\vartheta_{k}(x)=\sum_{i=1}^{J_{g, k}} w_{k}^{(i)} \mathrm{N}\left(x \mid \mathbf{m}_{9, k}^{(i)}, \mathbf{P}_{g, k}^{(i)}\right) .
$$

The most important contribution of this work is the modification of the state updating steps. According to (5), the target posterior PHD is

$$
D_{k}(x)=\left(1-p_{D, k}\right) D_{k \mid k-1}(x)+\sum_{z \in Z_{k}} D_{D, k}(x \mid z)
$$

where

$$
D_{D, k}(x \mid z)=\sum_{j=1}^{J_{k k-1}} w_{k}^{(j)} \operatorname{St}\left(x \mid \mathbf{m}_{k \mid k}^{(j)}, \mathbf{P}_{k \mid k}^{(j)}, v\right) .
$$

According to (5), (6), the states are assumed to follow Student's T distribution, thus $D_{D, k}(x \mid z)$ can be rewritten by a Gaussian form by setting an auxiliary random variable, i.e.,

$$
\begin{aligned}
D_{D, k}(x \mid z) \approx \sum_{j=1}^{J_{k k-1}} w_{k}^{(j)} \mathrm{N}\left(x \mid \mathbf{m}_{k \mid k}^{(j)}, \frac{1}{\lambda_{k}} \mathbf{P}_{k \mid k}^{(j)}\right) \\
\times \operatorname{Gamma}\left(\lambda_{k} \mid v / 2, v / 2\right) .
\end{aligned}
$$

Then, the states $\mathbf{m}_{k k k}^{(j)}$ and $\mathbf{P}_{k k k}^{(j)}$ can be updated by the TK filtering technique. The VB model approximates the nonlinear probability by an iteration process. For each iteration, the states will be calculated by

$$
\begin{gathered}
\mathbf{m}_{k \mid k}^{(j)}=\mathbf{m}_{k \mid k-1}^{(j)}+\mathbf{K}_{k}\left(z_{k}-\mathbf{H}_{k} \mathbf{m}_{k \mid k-1}^{(j)}\right), \\
\mathbf{P}_{k \mid k}^{(j)}=\mathbf{P}_{k \mid k-1}^{(j)}+\mathbf{K}_{k} \mathbf{S}_{k}^{-1} \mathbf{K}_{k}^{\mathrm{T}}
\end{gathered}
$$

where $\mathbf{H}_{k}$ is the observation matrix and set as $\mathbf{H}_{k}=\left[\begin{array}{llll}1 & 0 & 0 & 0 \\ 0 & 1 & 0 & 0\end{array}\right]$ in this scenario. The innovation parameters can be obtained by

$$
\begin{gathered}
\mathbf{K}_{k}=\left(\mathbf{P}_{k \mid k-1}^{(j)} \mathbf{H}_{k}^{\mathrm{T}}\right) \mathbf{S}_{k}^{-1}, \\
\mathbf{S}_{k}=\mathbf{H}_{k} \mathbf{P}_{k \mid k-1}^{(j)} \mathbf{H}_{k}^{\mathrm{T}}+\frac{1}{\bar{\lambda}_{k}} \mathbf{R}_{k}
\end{gathered}
$$

where $\bar{\lambda}_{k}$ is the expectation of the auxiliary random variable introduced in (11), and can be calculated by

$$
\begin{gathered}
\bar{\lambda}_{k}=(v+d) /\left(v+\bar{\gamma}_{k}\right), \\
\bar{\gamma}_{k}=\operatorname{tr}\left\{\left(\left(z_{k}-\mathbf{H}_{k} \mathbf{m}_{k \mid k}^{(j)}\right)\left(z_{k}-\mathbf{H}_{k} \mathbf{m}_{k \mid k}^{(j)}\right)^{\mathrm{T}}\right) \mathbf{R}_{k}^{-1}\right\} .
\end{gathered}
$$

The derivation process of (21) reference (28) and (32).

Given the number of iterations $N_{k}$, the states $\mathbf{m}_{k k k}^{(j)}$ and $\mathbf{P}^{(j)}{ }_{k k}$ will convergence to optimal value by iteration. According to (11), the updated target weight is

$$
w_{k}^{(j)}=\sum_{z \in Z_{k}} \frac{p_{D, k}(x) w_{k \mid k-1}^{(j)} \Psi_{k}^{(j)}\left(z \mid x, \lambda_{k}\right)}{\kappa_{k}(z)+p_{D, k} \sum_{\ell=1}^{J_{k k-1}} w_{k \mid k-1}^{(\ell)} \Psi_{k}^{(\ell)}\left(z \mid x, \lambda_{k}\right)}
$$


where the target likelihood $\Psi_{k}^{(j)}\left(z \mid x, \lambda_{k}\right)$ is difficult to solve, thus an approximate form will be given by

$$
\Psi_{k}^{(j)}\left(z \mid x_{k}, \lambda_{k}\right) \approx \phi_{k}^{(j)}\left(z \mid x_{k}\right) \varphi_{k}^{(j)}\left(z \mid \lambda_{k}\right) .
$$

According to section 2.1, the KL divergence method can be employed to solve $\phi_{k}^{(j)}\left(z \mid x_{k}\right)$ and $\varphi_{k}^{(j)}\left(z \mid \lambda_{k}\right)$. According to $[32$, p. 463$]$, thus we obtain

$$
\begin{aligned}
& \operatorname{KL}\left(\phi_{k}^{(j)}\left(z \mid x_{k}\right) \varphi_{k}^{(j)}\left(z \mid \lambda_{k}\right) \| \Psi_{k}^{(j)}\left(z \mid x_{k}, \lambda_{k}\right)\right) \\
& =\iint \phi_{k}^{(j)}\left(z \mid x_{k}\right) \varphi_{k}^{(j)}\left(z \mid \lambda_{k}\right) \\
& \quad \times \log \left(\frac{\phi_{k}^{(j)}\left(z \mid x_{k}\right) \varphi_{k}^{(j)}\left(z \mid \lambda_{k}\right)}{\Psi_{k}^{(j)}\left(z \mid x_{k}, \lambda_{k}\right)}\right) \mathrm{d} x_{k} \mathrm{~d} \lambda_{k} .
\end{aligned}
$$

According to (4), $\varphi_{k}^{(j)}\left(z \mid \lambda_{k}\right)$ and $\phi_{k}^{(j)}\left(z \mid x_{k}\right)$ become

$$
\begin{aligned}
& \log \left(\varphi_{k}^{(j)}\left(z \mid \lambda_{k}\right)\right)=E_{x_{k}}\left(\Psi_{k}^{(j)}\left(z \mid x_{k}, \lambda_{k}\right)\right)+\text { const },(25 a) \\
& \log \left(\phi_{k}^{(j)}\left(z \mid x_{k}\right)\right)=E_{\lambda_{k}}\left(\Psi_{k}^{(j)}\left(z \mid x_{k}, \lambda_{k}\right)\right)+\text { const } .(25 \mathrm{~b})
\end{aligned}
$$

According to formula (18), $\Psi_{k}^{(j)}\left(z \mid x_{k}, \lambda_{k}\right)$ is positively correlated with the distributions $\mathrm{N}\left(x \mid \mathbf{m}_{k \mid k}^{(j)}, \frac{1}{\lambda_{k}} \mathbf{P}_{k \mid k}^{(j)}\right)$ and $\operatorname{Gamma}\left(\lambda_{k} \mid v / 2, v / 2\right)$, i.e.

$$
\begin{aligned}
\Psi_{k}^{(j)}\left(z \mid x_{k}, \lambda_{k}\right) \propto \mathrm{N}\left(x_{k}, \mathbf{R}_{k} / \lambda_{k}\right) \\
\quad \times \mathrm{N}\left(\mathbf{m}_{k \mid k-1}, \mathbf{P}_{k \mid k-1}\right) \cdot \operatorname{Gamma}\left(\lambda_{k} \mid v / 2, v / 2\right) .
\end{aligned}
$$

Therefore,

$$
\begin{aligned}
& \log \left(\varphi_{k}^{(j)}\right.\left.\left(z \mid \lambda_{k}\right)\right)=E_{x_{k}}\left(\Psi_{k}^{(j)}\left(z \mid x_{k}, \lambda_{k}\right)\right)+\text { const } \\
& \propto E_{x_{k}}\left[\mathrm{~N}\left(x_{k}, \mathbf{R}_{k} / \lambda_{k}\right)+\mathrm{N}\left(\mathbf{m}_{k \mid k-1}, \mathbf{P}_{k \mid k-1}\right)\right. \\
&\left.+ \text { Gamma }\left(\lambda_{k} \mid \frac{v}{2}, \frac{v}{2}\right)\right]+ \text { const }
\end{aligned}
$$

where

$$
\begin{gathered}
E_{x_{k}}\left[\operatorname{Gamma}\left(\lambda_{k} \mid \frac{v}{2}, \frac{v}{2}\right)\right]=\left(\frac{v+d}{2}-1\right) \log \lambda_{k}-\frac{v \lambda_{k}}{2} \\
E_{x_{k}}\left(\mathrm{~N}\left(x_{k}, \mathbf{R}_{k} / \lambda_{k}\right)\right)=-\frac{1}{2} \bar{\lambda}_{k} \bar{\gamma}_{k} \\
\bar{\gamma}_{k}=\operatorname{tr}\left\{\left(\left(z_{k}-\mathbf{H}_{k} \mathbf{m}_{k}\right)\left(z_{k}-\mathbf{H}_{k} \mathbf{m}_{k}\right)^{\mathrm{T}}\right) \mathbf{R}_{k}^{-1}\right\}
\end{gathered}
$$

Combining formulas (27), (28), we obtain

$$
\varphi_{k}^{(j)}\left(z \mid \lambda_{k}\right) \propto \exp \left(\left(\frac{v+d}{2}-1\right) \log \bar{\lambda}_{k}-\frac{v+\bar{\gamma}_{k}}{2} \bar{\lambda}_{k}\right) .
$$

Similarly, $\phi_{k}^{(j)}\left(z \mid x_{k}\right)$ can be written as

$$
\begin{aligned}
\log \left(\phi_{k}^{(j)}\left(z \mid x_{k}\right)\right)=E_{\lambda_{k}}\left(\Psi_{k}^{(j)}\left(z \mid x_{k}, \lambda_{k}\right)\right)+\mathrm{const} \\
\propto E_{\lambda_{k}}\left[\mathrm{~N}\left(x_{k}, \mathbf{R}_{k} / \lambda_{k}\right)+\mathrm{N}\left(\mathbf{m}_{k \mid k-1}, \mathbf{P}_{k \mid k-1}\right)\right. \\
\left.+\operatorname{Gamma}\left(\lambda_{k} \mid v / 2, v / 2\right)\right]+\mathrm{const} \\
\propto-\frac{1}{2} \lambda_{k}\left(z_{k}-\mathbf{H}_{k} \mathbf{m}_{k \mid k}^{(j)}\right)^{\mathrm{T}} \mathbf{R}_{k}^{-1}\left(z_{k}-\mathbf{H}_{k} \mathbf{m}_{k \mid k}^{(j)}\right) \\
-\frac{1}{2}\left(\mathbf{m}_{k \mid k}^{(j)}-\mathbf{m}_{k \mid k-1}^{(j)}\right)^{\mathrm{T}}\left(\mathbf{P}_{k \mid k-1}^{(j)}\right)\left(\mathbf{m}_{k \mid k}^{(j)}-\mathbf{m}_{k \mid k-1}^{(j)}\right) .
\end{aligned}
$$

Thus we can obtain

$$
\begin{aligned}
\phi_{k}^{(j)}\left(z \mid x_{k}\right) \propto \\
\quad \exp \left(-\frac{1}{2} \lambda_{k}\left(z_{k}-\mathbf{H}_{k} \mathbf{m}_{k \mid k}^{(j)}\right)^{\mathrm{T}} \mathbf{R}_{k}^{-1}\left(z_{k}-\mathbf{H}_{k} \mathbf{m}_{k \mid k}^{(j)}\right)\right. \\
\left.\quad-\frac{1}{2}\left(\mathbf{m}_{k \mid k}^{(j)}-\mathbf{m}_{k \mid k-1}^{(j)}\right)^{\mathrm{T}}\left(\mathbf{P}_{k \mid k-1}^{(j)}\right)\left(\mathbf{m}_{k \mid k}^{(j)}-\mathbf{m}_{k \mid k-1}^{(j)}\right)\right)
\end{aligned}
$$

Note that, the expectation $\mathrm{E}\left(\lambda_{k}\right)$ is not the expectation of $\operatorname{Gamma}(v / 2, v / 2)$, and it is the expectation of $\varphi_{k}^{(j)}\left(z \mid \lambda_{k}\right)$. Therefore, it is easy to be obtained that

$$
\bar{\lambda}_{k}=E\left(\varphi_{k}^{(j)}\left(z \mid \lambda_{k}\right)\right)=(v+d) /(v+\bar{\gamma})_{k} .
$$

Note that the formulas (19)-(21) determine the update of state $\mathbf{m}_{k k k}^{(j)}$ and $\mathbf{P}_{k \mid k}^{(j)}$ in the tracking process. These formulas are based on TK filtering technology and correspond to formulas (8), (9). The weight of each component can be calculated by formula (22). The formula (23) is the likelihood function used in (22). Formulas (29) and (31) are final derived results for solving (23), and (24)-(28), and (30) are its derivation process. The Pseudocode is shown in Tab. 1. The code can be available at [31].

In order to ensure that the calculation amount of a PHD filter is not too large, similar components need to be merged, and the components with small weight need to be pruned. The merging, pruning steps of the proposed TKPHD filter are the same as the standard PHD filter, and the details can be found in [4]. Note that the above proposed method mainly modifies the state filtering step and the likelihood function, that is, it can be directly used in the CPHD framework. Therefore, for brevity and readability, the formulas of the CPHD filter won't be listed here.

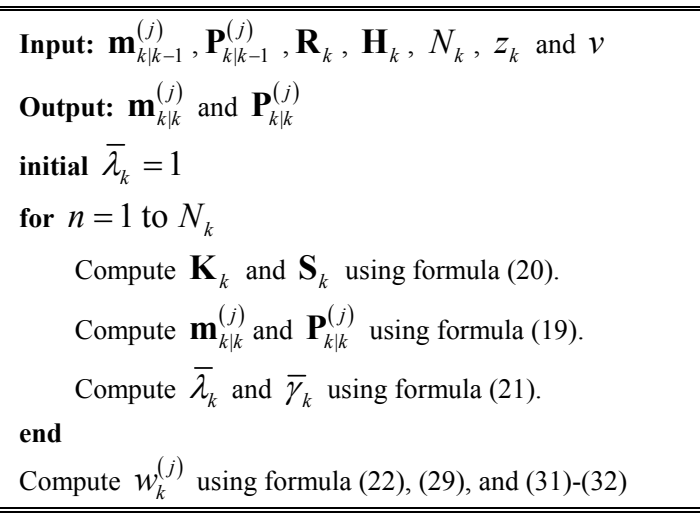

Tab. 1. Pseudo-code for TK-PHD filter's updating step. 


\subsection{The Implementation for BAR Scenarios}

Assuming that the measurement model is formed as $z_{k}=\left\{\theta_{k}, \rho_{k}\right\}$, where $\theta_{k}$ and $\rho_{k}$ are the bearing and the range values, respectively. The linear target state is modeled by $x_{k}=\left\{u_{\mathrm{x}}, u_{\mathrm{y}}, v_{\mathrm{x}}, v_{\mathrm{y}}\right\}$ where $u_{\mathrm{x}}$ and $u_{\mathrm{y}}$ are the two-dimensional Cartesian coordinate vectors with corresponding velocity vectors $v_{\mathrm{x}}$ and $v_{\mathrm{y}}$. Obviously, the major problem of BAR scenarios is how to filter with different types of data. A simple solution is to map the BAR measurements to Cartesian coordinates. However, the mapped measurements is a non-linear form, thus the tracking accuracy of a linear PHD filter will decrease. In order to solve this problem, Vo et al. presented the EK-PHD filter using EK method in [4]. In this section, combining the EK method, the extended TK-PHD (E-TK-PHD) filter is proposed for the BAR scenarios.

Assuming that the observation is a non-linear model:

$$
\begin{gathered}
x_{k}=f_{k}\left(x_{k-1}, \varsigma_{k-1}\right), \\
z_{k}=h_{k}\left(x_{k}, \varepsilon_{k}\right)
\end{gathered}
$$

where $\zeta_{k-1}$ and $\varepsilon_{k}$ are zero-mean Gaussian process noise and measurement noise with covariances $\mathbf{Q}_{k-1}$ and $\mathbf{R}_{k}$, respectively. The Jacobian-matrix based local linearization method of the EK method can be employed to the proposed method. Considering the BAR measurements, the observation model can be written as

$$
h_{k}\left(x_{k}, \varsigma_{k}\right)=\left[\begin{array}{c}
\arctan \left(u_{\mathrm{y}} / u_{\mathrm{x}}\right) \\
\sqrt{u_{\mathrm{x}}^{2}+u_{\mathrm{y}}^{2}}
\end{array}\right]+\varsigma_{k}
$$

The state transition model matrix is still the linear form, thus $\mathbf{F}_{k}$ in (13b) does not change. The major steps of the E-TK-PHD filter are the same as the TK-PHD filter. However, the observation matrix in (20a) becomes

$$
\begin{aligned}
\hat{\mathbf{H}}_{k} & =\partial h_{k}\left(x_{k}, 0\right) /\left.\partial x_{k}\right|_{x_{k}=m_{k k-1}^{(j)}} \\
& =\left[\begin{array}{llll}
u_{\mathrm{y}} /\left(u_{\mathrm{x}}^{2}+u_{\mathrm{y}}^{2}\right) & u_{\mathrm{x}} /\left(u_{\mathrm{x}}^{2}+u_{\mathrm{y}}^{2}\right) & 0 & 0 \\
u_{\mathrm{x}} / \sqrt{u_{\mathrm{x}}^{2}+u_{\mathrm{y}}^{2}} & u_{\mathrm{y}} / \sqrt{u_{\mathrm{x}}^{2}+u_{\mathrm{y}}^{2}} & 0 & 0
\end{array}\right] .
\end{aligned}
$$

\section{Simulation Results}

This section shows the performances between proposed methods and other important tracking methods. There are two target track models shown in Fig. 1(a) and (b). The probability of measurement-outliers at each scan is $10 \%$. In order to verify the performance of the proposed filters, three different scenarios are tested respectively, and the parameters are shown in Tab. 2. In BAR scenarios, $\sigma_{\theta}{ }^{2}$

\begin{tabular}{|c|c|c|c|}
\hline & Scenario 1 & Scenario 3 & Scenario 3 \\
\hline Linear/BAR & Linear & BAR & BAR \\
\hline $\begin{array}{c}\text { Measurement } \\
\text { noise }\end{array}$ & $\mathbf{R}_{k}=\left[\begin{array}{cc}10^{2} & 0 \\
0 & 10^{2}\end{array}\right]$ & $\begin{array}{c}\sigma_{\theta}^{2}=(\pi / 90)^{2} \\
\sigma_{\rho}^{2}=10^{2}\end{array}$ & $\begin{array}{c}\sigma_{\theta}^{2}=(\pi / 90)^{2} \\
\sigma_{\rho}^{2}=10^{2}\end{array}$ \\
\hline Outliers noise & $\mathbf{R}_{k}=\left[\begin{array}{cc}50^{2} & 0 \\
0 & 50^{2}\end{array}\right.$ & No outliers & $\begin{array}{c}\sigma_{\theta}^{2}=(\pi / 20)^{2} \\
\sigma_{\rho}^{2}=40^{2}\end{array}$ \\
\hline $\begin{array}{l}\text { Surveillance } \\
\text { volume }\left(\mathrm{m}^{2}\right) \\
\end{array}$ & $\mathcal{S}=2000 \times 2000$ & $\mathcal{S}=4000 \times 2000$ & $\mathcal{S}=4000 \times 2000$ \\
\hline $\begin{array}{l}\text { Clutters' } \\
\text { intensity }\end{array}$ & $\kappa_{k}=2.5 \times 10^{-6}$ & $\kappa_{k}=1.25 \times 10^{-6}$ & $\kappa_{k}=1.25 \times 10^{-6}$ \\
\hline $\begin{array}{l}\text { Scanning } \\
\text { interval (s) }\end{array}$ & $T_{\mathrm{s}}=1$ & $T_{\mathrm{s}}=1$ & $T_{\mathrm{s}}=1$ \\
\hline Process noise & $\mathbf{Q}_{k}=\left[\begin{array}{ll}1 & 0 \\
0 & 1\end{array}\right]$ & $\mathbf{Q}_{k}=\left[\begin{array}{ll}1 & 0 \\
0 & 1\end{array}\right]$ & $\mathbf{Q}_{k}=\left[\begin{array}{ll}1 & 0 \\
0 & 1\end{array}\right]$ \\
\hline $\begin{array}{c}\text { Survival } \\
\text { probability }\end{array}$ & $p_{\mathrm{S}}=0.99$ & $p_{\mathrm{S}}=0.99$ & $p_{\mathrm{S}}=0.99$ \\
\hline $\begin{array}{l}\text { Detection } \\
\text { probability }\end{array}$ & $p_{\mathrm{D}}=0.99$ & $p_{\mathrm{D}}=0.99$ & $p_{\mathrm{D}}=0.99$ \\
\hline
\end{tabular}
and $\sigma_{\rho}^{2}$ denote the bearing and range noise, respectively.

Note that: 1) Scenario 1 is set to show the performance of TK-PHD/CPHD filters in the linear cases. 2) Scenario 2 is a non-linear case without measurement-
Tab. 2. Parameters of three scenarios.

\begin{tabular}{|c|c|c|}
\hline Kinematical state & Weight & Error covariance \\
\hline $\mathbf{m}_{0}=\left[u_{\mathrm{x}, 0}, u_{\mathrm{y}, 0}, 0,0\right]^{\mathrm{T}}$ & $w_{0}=0.01$ & $\mathbf{P}_{0}=\operatorname{diag}\left(\left[5^{2}, 5^{2}, 10^{2}, 10^{2}\right]\right)$ \\
\hline
\end{tabular}

Tab. 3. Initial parameters of each component.

outliers. The most important purpose of the proposed filters is to deal with the measurement-outliers, thus this scenario can well test the robustness of the proposed filters. 3) Scenario 3 is the most complex one, which can show the effectiveness of the proposed filters.

The initial parameters of each component are shown in Tab. 3 .

The proposed methods are compared with: standard GM-PHD [4]/CPHD [7] filters; EK-PHD [4] filter; JointLMB (J-LMB) [13] filter (an important and advanced filter in recent years) and its form of directly using EK (called EK-J-LMB filter). Note that except for EK-PHD, EK-JLMB and E-TK-PHD filters, other filters cannot handle the BAR measurement directly, thus the BAR measurements are converted into Cartesian coordinates for these filters. The given number of recurrences is $N_{k}=10$. The degree of freedom of Student's T distribution is $v=7$ with linear model and $v=15$ with BAR model. 200 Monte Carlo simulations were preformed, and the performances are presented in terms of the OSPA metric [29] and computational time cost. The simulation environment is as follows: Software: MATLAB 2019(a); Computer system: Windows 10; CPU: Intel(R) Core(TM) i7-6700HQ.

\subsection{Scenario 1}

In this scenario, there are four targets moving in a straight line. Two of them cross at the 50th scan, and their tracks are shown in Fig. 1(a). Figure 2 shows the average performances of 200 Monte Carlo runs. 


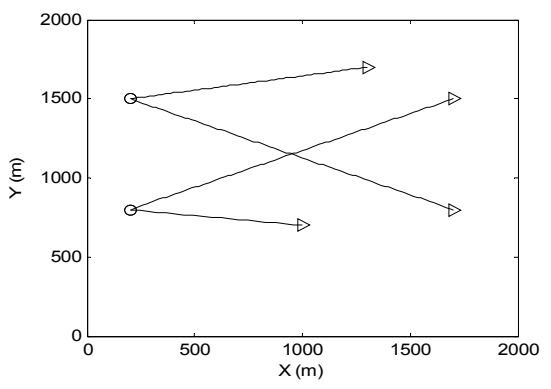

(a) Target track model 1

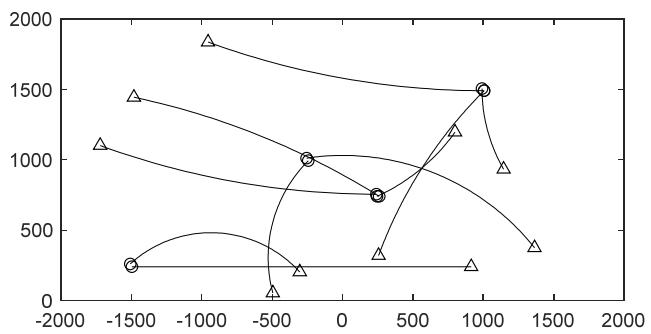

(b) Target track model 2

Fig. 1. Target track models. Circles and triangles denote the target starting and ending positions, respectively.

Figure 2(a) shows the average OSPA values, and the main conclusions are summarized as follows: 1) The OSPA values of standard EK-PHD and GM-PHD/CPHD filters are the largest, which shows that their performances are worse than other filters. 2) The OSPA values of TK-CPHD filter is lower than the J-LMB filter, which shows that the proposed TK-CPHD filter has the best performance in the linear scenarios with measurement-outliers.

Note that, the OSPA values of J-LMB filter and TKCPHD filter are roughly the same from 40-60 s (when the two targets are closely spaced). The reason is that the tracking accuracy of the standard LMB framework is higher than the standard PHD/CPHD framework when targets are closely spaced. Thus, this accuracy difference offsets the accuracy advantage of TK-CPHD filter when targets are closely spaced.

Figure 2(b) shows the average estimated number of targets. The performance of J-LMB and TK-PHD filters are roughly the same. Compared with the results of Fig. 2(a), it mains that the J-LMB filter has the highest position estimation accuracy than other filters. The estimated values of the proposed TK-CPHD filter are obviously closest to the true values. It indicates that the TKCPHD filter has better performance advantages in cardinalization estimation.

Figure 2(c) shows the average computational time costs. In this linear scenario, obviously, the computational complexity of the PHD framework is the smallest, CPHD is the second, and LMB is the largest. Moreover, combined with the results in Fig. 2(a) and (b), the proposed TKCPHD filter achieves higher accuracy with less time cost than J-LMB filter.

Figure 3 shows the average OSPA values of the whole tracking process with different detection probability

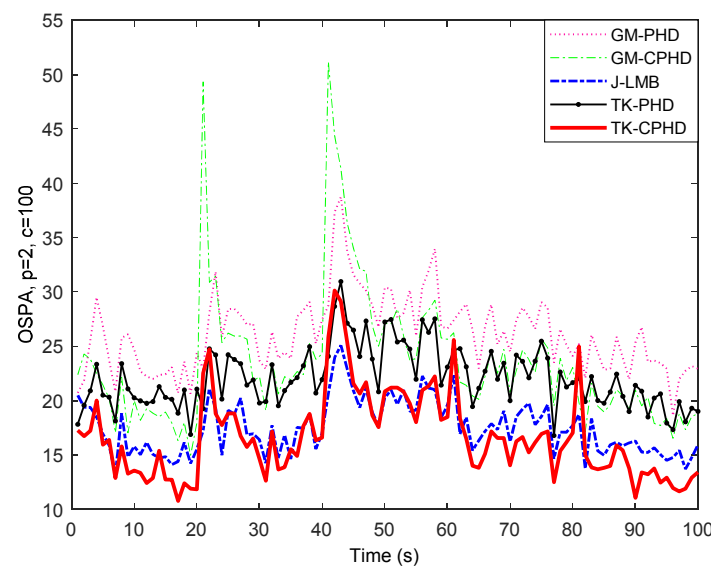

(a) Average OSPA values

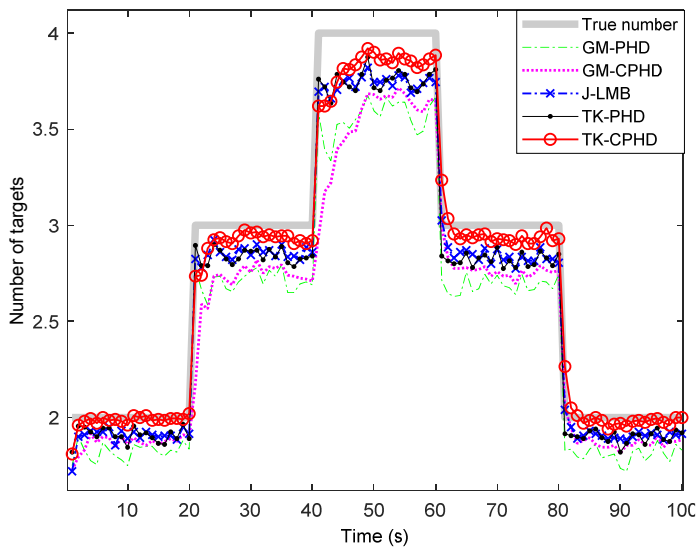

(b) Average number of targets

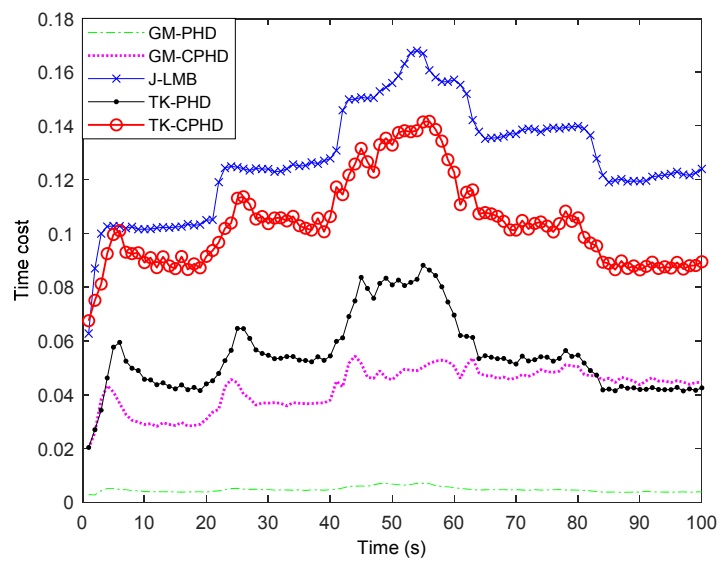

(c) Average computational time costs

Fig. 2. Average performances of different filters in Scenario 1.

$p_{D}$ in 200 Monte Carlo runs. The proposed TK-CPHD filter achieves the better performance than the J-LMB filter when $p_{D}=0.99$. However, when $p_{D}=0.95$, the proposed method has only a slight accuracy advantage. When $p_{D}<0.95$, the J-LMB filter achieves the best performance. Therefore, the proposed method is suitable for the observation system with high detection rate. The reason for this is that the LRFS-based filter considers the track information to handle the target missed detection. Thus it is hard for a RFS-based filter to be more accurate than the J-LMB filter in a low detection probability scenario. However, Figure 3 also shows that the proposed TK-CPHD filter can 


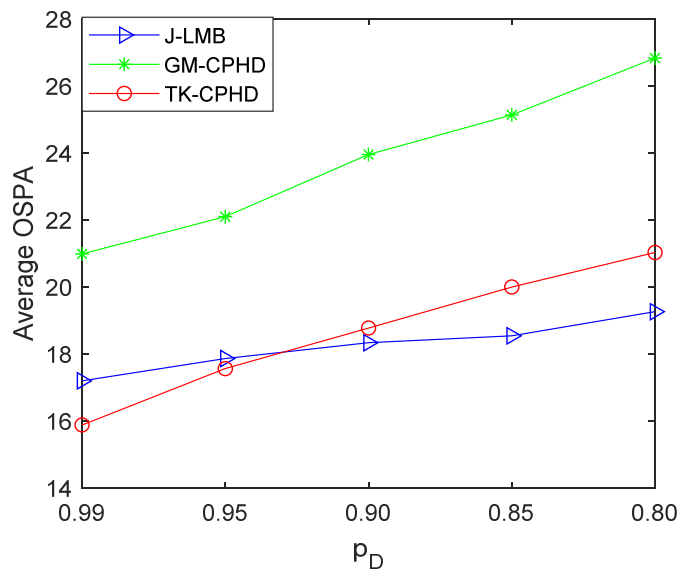

Fig. 3. Average OSPA values with different $p_{D}$.

greatly improve the accuracy compared with the CPHD filter with any $p_{D}$. Therefore, applying the proposed idea to LRFS may solve this problem, but further derivation of VB-based track likelihood is required.

\subsection{Scenario 2}

Ten targets and the tracks of Scenario 2 were shown in Fig. 1(b). Figure 4 shows the average performances of 200 Monte Carlo runs.

Figure 4(a) shows the average OSPA values, and the conclusions are as follows: 1) In Fig. 2(a), the precision of the J-LMB filter is higher than that of proposed TK-PHD filter, but in Fig. 4.(a), their precisions are similar, which shows that the proposed TK method is robust to nonlinear scenario. 2) The accuracy of E-TK-PHD is similar to that of EK-PHD, and both are higher than other standard filters except the EK-J-LMB. 3) Due to the accurate track association, the performance of the EK-J-LMB filter is better than other filters when the targets are closely-spaced.

Figure 4(b) shows the average estimated number of targets. It can be seen that the main reason for the decrease of accuracy of the J-LMB filter is the inaccuracy of the target number estimation. However, the EK-J-LMB filter can achieve the best performance, which leads to the best performance of the OSPA results in Fig. 3(a).

Figure 4(c) shows the average computational time costs. As can be seen, the time cost of the proposed E-TKPHD filter is the largest, while that of the EK-J-LMB filter is smaller. Combined with the results of Fig. 4(a) and (b), it shows that the performance of EK-J-LMB filter is the best in this non-linear scenario.

\subsection{Scenario 3}

In this scenario, the performances of the three EKbased filters are compared, and target tracks are the same as Scenario 2. The difference between the two scenarios is that the sensor can generate randomly measurementoutliers. Figure 4 shows the average performances of 200 Monte Carlo runs.

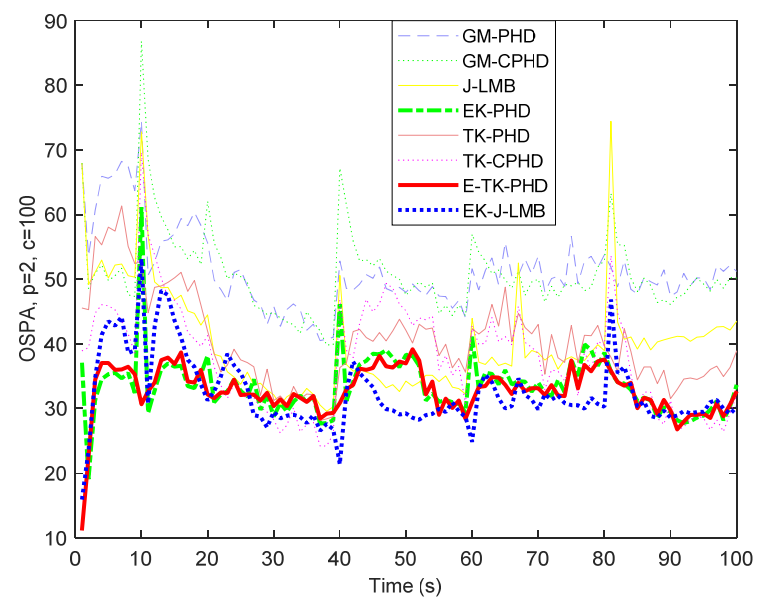

(a) Average OSPA values

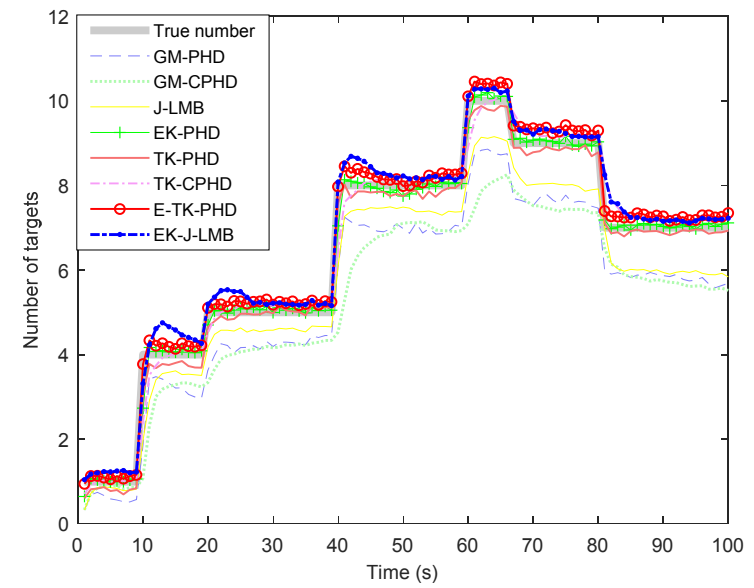

(b) Average number of targets

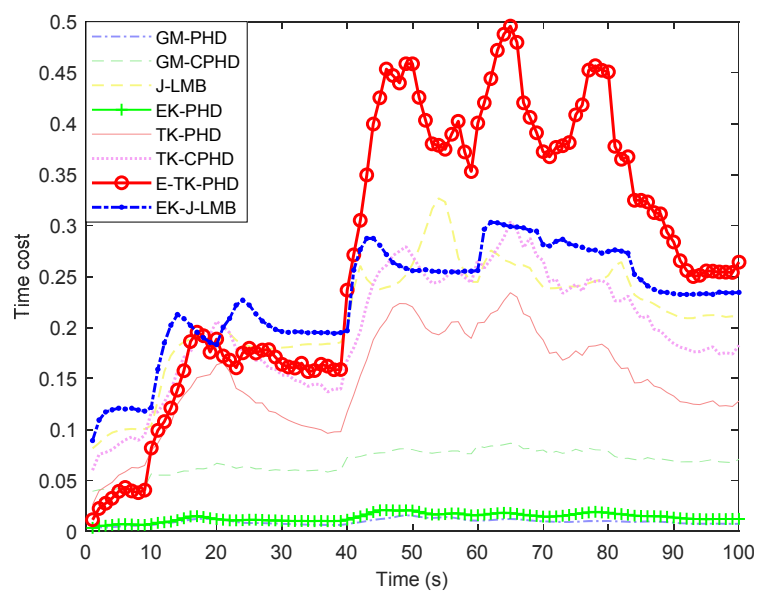

(c) Average computational time costs

Fig. 4. Average performances of different filters in Scenario 2.

Figure 5(a) shows the average OSPA values. In this complex scenario with nonlinear and measurement-outliers, the TK-PHD/CPHD filters achieve better performance than GM-PHD/CPHD and J-LMB filters. Based on the fact that none of these filters use EK technology, it means that proposed filters have strong robustness in dealing with complex scenarios. Moreover, the proposed E-TK-PHD filter 
achieves the best performance, which shows the effectiveness of this work. Note that the performance of the EK-JLMB filter is slightly better than E-TK-PHD filter, and the reason is still its accurate track association. Figure 5(b) shows the average estimated number of targets, which shows that the proposed E-TK-PHD filter performs best.

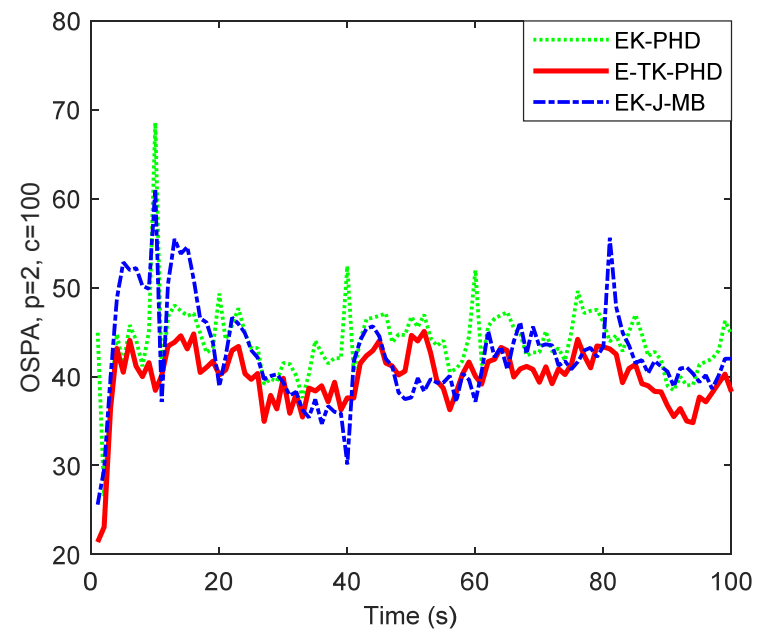

(a) Average OSPA values.

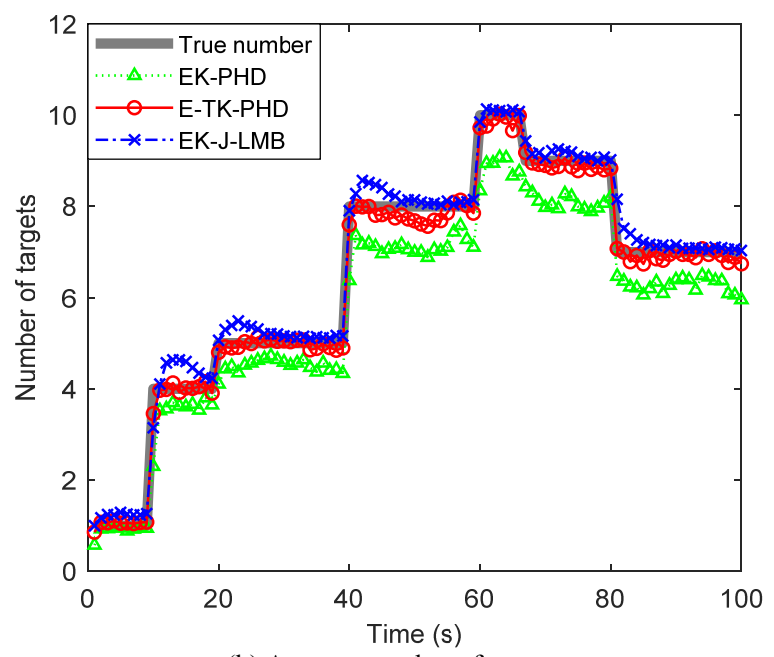

(b) Average number of targets.

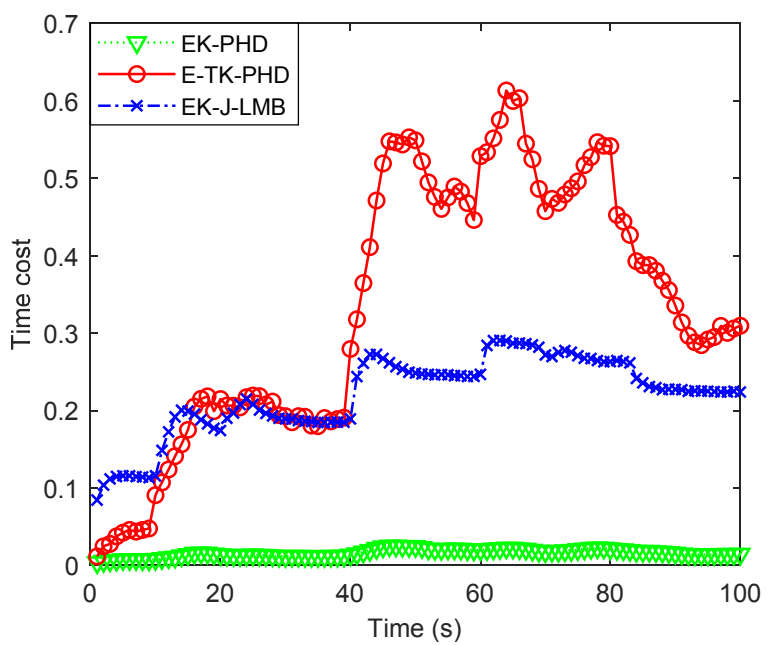

(c) Average computational time costs

Fig. 5. Average performances of different filters in Scenario 3.
Figure 5(c) shows the average computational time costs. As can be seen, the time cost of the proposed E-TKPHD filter is the highest, thus the balance of precision and time cost should be considered in practical application.

\section{Conclusions}

This paper proposed robust PHD/CPHD filters to deal with the measurement-outlier and BAR problems based on TK filtering technique and VB method. The measurement noise is assumed to follow the Student's T distribution, thus target states are updated by employing the BV based TK filtering technique. Moreover, the posterior likelihood is approximated by the VB framework, thus the proposed filters will have stronger robustness to deal with non-linear scenarios, especially measurement-outlier scenarios.

The proposed methods not only can be applied to PHD and CPHD filters, but also has the potential to be applied to the LRFS framework, such as the J-LMB filter [13]. The J-LMB filter shows better performance than standard PHD filters, thus the use of the proposed methods for this filter can further improve the tracking accuracy theoretically. Moreover, we plan to apply the proposed method to extended target PHD filters, e.g. the modified Gaussian inverse Wishart PHD filter [30]. Extended target PHD filters require partitioning the measurement set each scan, and partition error is a common measurement-outliers problem. Therefore, the use of TK filtering method can theoretically improve tracking accuracy of an extended target PHD filter.

\section{Acknowledgments}

This paper is supported by the National Natural Science Foundation of China (No. 61806006, 61902160, 61806088); the Natural Science Foundation of the Jiangsu Higher Education Institutions of China (No. 19KJD510003, 19KJB520006); Natural Science Research Project of Colleges and Universities in Anhui Province (No. KJ2018A0083); the foundation of Changzhou Science and Technology Plan (Applied Basic Research) (No. CJ20190076).

\section{References}

[1] MAHLER, R. Multi-target Bayes filtering via first-order multitarget moments. IEEE Transactions on Aerospace and Electronic Systems, 2003, vol. 39, no. 4, p. 1152-1178. DOI: 10.1109/TAES.2003.1261119

[2] VO, B. T., VO, B. N. Labeled random finite sets and multi-object conjugate priors. IEEE Transactions on Signal Processing, 2013, vol. 61 , no. 13 , p. $3460-3475$. DOI: $10.1109 /$ tsp.2013.2259822

[3] VO, B. N., VO, B. T., PHUNG, D. Labeled random finite sets and the Bayes multi-target tracking filter. IEEE Transactions on Signal Processing, 2014, vol. 62, no. 24, p. 6554-6567. DOI: $10.1109 /$ tsp.2014.2364014 
[4] REUTER, S., VO, B. T., VO, B. N., et al. The labeled multiBernoulli filter. IEEE Transactions on Signal Processing, 2014, vol. 62, no. 12, p. 3246-3260. DOI: 10.1109/tsp.2014.2323064

[5] VO, B. N., MA, W. K. The Gaussian mixture probability hypothesis density filter. IEEE Transactions on Signal Processing, 2010, vol. 54, no. 11, p. 4091-4104. DOI: 10.1109/TSP.2006.881190

[6] MAHLER, R. A theory of PHD filters of higher order in target number. In Proceedings of the International Society for Optical Engineering. Lockheed Martin Corp, USA, May 2006, p. 1-12. DOI: $10.1117 / 12.667083$

[7] MAHLER, R. PHD filters of higher order in target number. IEEE Transactions on Aerospace and Electronic Systems, 2007, vol. 43, no. 4 , p. $1523-1543$. DOI: $10.1109 /$ taes.2007.4441756

[8] VO, B. T., VO, B. N., CANTONI, A. Analytic implementations of the cardinalized probability hypothesis density filter. IEEE Transactions on Signal Processing, 2007, vol. 55, no. 7, p. 3553-3567. DOI: $10.1109 /$ tsp.2007.894241

[9] MAHLER, R., VO, B. T., VO, B. N. CPHD filtering with unknown clutter rate and detection profile. IEEE Transactions on Signal Processing, 2011, vol. 59, no. 8, p. 3497-3513. DOI: $10.1109 /$ tsp.2011.2128316

[10] BRYANT, D. S., DELANDE, E. D., GEHLY, S., et al. The CPHD filter with target spawning. IEEE Transactions on Signal Processing, 2017, vol. 65, no. 5, p. 13124-13138. DOI: $10.1109 /$ tsp.2016.2597126

[11] CLARK, D. E., PANTA, K., VO, B. N. The GM-PHD filter multiple target tracker. In Proceedings of the International Conference on Information Fusion. Florence (Italy), 2006, p. 1-8. DOI: 10.1109/ICIF.2006.301809

[12] PANTA, K., CLARK, D. E., VO, B. N. Data association and track management for the Gaussian mixture probability hypothesis density filter. IEEE Transactions on Aerospace and Electronic Systems, 2009, vol. 45, no. 3, p. 1003-1016. DOI: 10.1109/TAES.2009.5259179

[13] VO, B. N., VO, B. T., HOANG, H. G. An efficient implementation of the generalized labeled multi-Bernoulli filter. IEEE Transactions on Signal Processing, 2017, vol. 65, no. 8, p. 1975-1987. DOI: 10.1109/TSP.2016.2641392

[14] YANG, B., WANG, J., WANG, W. An efficient approximate implementation for labeled random finite set filtering. Signal Processing, 2018, vol. 150, p. 215-227. DOI: 10.1016/j.sigpro.2018.04.015

[15] PAPI, F., VO, B. N., VO, B. T., et al. Generalized labeled multiBernoulli approximation of multi-object densities. IEEE Transactions on Signal Processing, 2015, vol. 63, no. 20, p. 5487-5497. DOI: $10.1109 /$ TSP.2015.2454478

[16] JAZWINSKI, A. H. Stochastic Processes and Filtering Theory. New York: Academic Press, 1970. ISBN: 9780123815507

[17] JULIER, S., UHLMANN, J. Unscented filtering and nonlinear estimation. Proceedings of the IEEE, 2004, vol. 92, no. 3, p. 401 to 422. DOI: $10.1109 /$ JPROC.2003.823141

[18] VO, B. N., SINGH, S., DOUCET, A. Sequential Monte Carlo methods for multitarget filtering with random finite sets. IEEE Transactions on Aerospace and Electronic Systems, 2005, vol. 41, no. 4, p. 1224-1245. DOI: 10.1109/taes.2005.1561884

[19] WHITELEY, N., SINGH, S., GODSILL, S. Auxiliary particle implementation of probability hypothesis density filter. IEEE Transactions on Aerospace and Electronic Systems, 2010, vol. 46, no. 3, p. 1437-1454. DOI: 10.1109/taes.2010.5545199

[20] XIE, Y., SONG, T. L. Bearings-only multi-target tracking using an improved labeled multi-Bernoulli filter. Signal Processing, 2018, vol. 151, p. 32-44. DOI: 10.1016/j.sigpro.2018.04.027
[21] SU, Z., JI, H., ZHANG, Y. An improved measurement-oriented marginal multi-Bernoulli/Poisson filter. Radioengineering, 2019, vol. 28, no.1, p. 191-198. DOI: 10.13164/re.2019.0191

[22] UCAR, M. B., YILMAZ, D. A new motion model selection approach for multi-model particle filters. Radioengineering, 2019, vol. 28 , no. 4, p. 793-800. DOI: 10.13164/re.2019.0793

[23] HUANG, Y., ZHANG, Y., LI, N., et al. A novel robust Student's T-based Kalman filter. IEEE Transactions on Aerospace and Electronic Systems, 2017, vol. 53, no. 3, p. 1545-1554. DOI: 10.1109/TAES.2017.2651684

[24] HUANG, Y., ZHANG, Y., ZHAO, Y., et al. A novel robust Gaussian-Student's $\mathrm{T}$ mixture distribution based Kalman filter. IEEE Transactions on Signal Processing, 2019, vol. 67, no. 13, p. 3606-3620. DOI: 10.1109/TSP.2019.2916755

[25] LIU, Z., CHEN, S., WU, H., et al. A Student's T mixture probability hypothesis density filter for multi-target tracking with outliers. Sensors, 2018, vol. 18, no. 4, p. 1-23. DOI: $10.3390 / \mathrm{s} 18041095$

[26] PICHE, R., SARKKA, S., HARTIKAINEN, J. Recursive outlierrobust filtering and smoothing for nonlinear systems using the multivariate Student-T distribution. In Proceedings of the 2012 IEEE International Workshop on Machine Learning for Signal Processing. Santander (Spain), 2012, p. 1-8. DOI: 10.1109/MLSP.2012.6349794

[27] WATANABE, S., MINAMI, Y., NAKAMURA, A., et al. Variational Bayesian estimation and clustering for speech recognition. IEEE Transactions on Speech and Audio Processing, 2004, vol. 12, no. 4, p. 365-381. DOI: 10.1109/TSA.2004.828640

[28] KULLBACK, S., LEIBLER, R. On information and sufficiency. The Annals of Mathematical Statistics, 1951, vol. 22, no. 1, p. 79 to 86. DOI: $10.1214 / \mathrm{aoms} / 1177729694$

[29] SCHUHMACHER, D., VO, B. T., VO, B. N. A consistent metric for performance evaluation of multi-object filters. IEEE Transactions on Signal Processing, 2008, vol. 56, no. 8, p. 3447 to 3457. DOI: $10.1109 /$ tsp.2008.920469

[30] PENG LI, HONGWEI GE, JINLONG, YANG. Adaptive measurement partitioning algorithm for a Gaussian inverse Wishart PHD filter that tracks closely spaced extended targets. Radioengineering, 2017, vol. 26, no. 2, p. 573-580. DOI: $10.13164 / \mathrm{re} .2017 .0573$

[31] PENG LI. Code of TK-PHD/CPHD filter. Available at: https://github.com/lptiancai/TK-PHD-filter.git

[32] BISHOP, C. M. Pattern Recognition and Machine Learning. New York (USA): Springer Verlag, 2006. ISBN 978-0-387-31073-2

\section{About the Authors ...}

Peng LI was born in 1989. He received his B.S. degree in Mathematics and Applied Mathematics from Langfang Normal University. He received his Ph.D. degree in Control Science and Engineering from Jiangnan University. Currently, he works at Jiangsu University of Technology. His research interests include target tracking and signal processing.

Chen XU (corresponding author) was born in 1990. He received his Ph.D. degree in Control Science and Engineering from Jiangnan University. Currently, he works at Jiangnan University. His research interests include fault detection and signal processing. 
Wenhui WANG was born in 1990. She received her M.S. degree in Design Science from Jiangnan University. Her research interest is target tracking and signal processing.
Shuzhi SU was born in 1987. He received his Ph.D. degree in Control Science and Engineering from Jiangnan University. His research interests are image recognition, canonical correlation analysis and signal processing. 